\title{
Predictive Value of CD229, CD319 and c-Maf Overexpression for Treatment Response in Multiple Myeloma Patients
}

\author{
Huda F Ebian ${ }^{1}$, Mai Abdelwahab*2, Samah S Elbasateeny ${ }^{2}$, Shimaa Abdelmoneem ${ }^{3}$, Heba E Abd Elrahman ${ }^{1}$ \\ Departments of ${ }^{1}$ Clinical Pathology, ${ }^{2}$ Pathology and ${ }^{3}$ Internal Medicine, \\ Hematology Unit, Faculty of Medicine, Zagazig University, Egypt. \\ *Corresponding author: Mai Abdelwahab, Mobile: (+20) 01003149432, E-Mail: Maiabdelwahab@ymail.com
}

\begin{abstract}
Background: Ly-9 (CD229) and SLAMF7 (CD319) are more stable markers that could be used to replace the less stable conventional markers CD138 and CD38 in multiple myeloma (MM) patient follow-up. In those patients, the correlation between these markers and the histopathologic marker c-Maf has not been well investigated.

Objective: To assess the predictive value of CD229, CD319, and c-MAF overexpression on outcome of treatment in MM patients.

Patients and Methods: 61 newly diagnosed multiple myeloma patients were involved in this prospective study. Flow cytometric analysis of bone marrow (BM) samples for CD229 PE, CD319 PE, CD138 PerCP and CD38FITC as well as BM biopsy immunohistochemically were analyzed for c-Maf expression

Results: CD229 and CD319 were highly expressed in ( $\geq 93.95 \%)$ and ( $\geq 95.6 \%)$ of MM patients respectively. c-Maf was positive in (29.5\%) of patients. In MM patients with high expression of CD229 and CD319 as well as those with c-Maf positivity showed high serum $\mathrm{Ca}^{++}, \beta 2 \mathrm{M}, \mathrm{CD} 138$ and CD38. CD229 was significantly correlated with CD319, CD38, $\mathrm{CD} 138, \beta 2 \mathrm{M}$ and serum $\mathrm{Ca}$ (p-values were $<0.001,0.01,0.01,0.009$, and 0.02 respectively). Patients with overexpression of CD 229, CD319 and c-Maf were more refractory to treatment (p-values were $<0.001,<0.001$ and $<0.001$ respectively).

Conclusion: We detected that both CD229 and CD319 were significantly overexpressed on MM cells and correlated significantly with CD138 and CD38, suggesting their use as alternative markers for MM diagnosis as well as followup. Patients with CD229,CD319, and c-Maf overexpression were associated with a significant poor response to therapy. Keywords: CD229, CD319, c-Maf, MM, SLAMF7.
\end{abstract}

\section{INTRODUCTION}

Multiple myeloma (MM) is a malignant neoplasm of plasma cells that accumulate in bone marrow, and lead to destruction of bone then bone marrow failure. The primary work up for diagnosis should include a complete history, physical examination and the following basic blood studies: CBC with differential and platelet counts; serum creatinine; blood urea nitrogen (BUN); serum electrolytes and calcium; albumin; $\beta 2$-microglobulin and lactate dehydrogenase (LDH). Increased creatinine and BUN indicate decreased renal functions, while LDH levels help to evaluate tumor cell burden in cases of lymphoma-like or plasmablastic myeloma ${ }^{(\mathbf{1})}$.

Multiple myeloma (MM) is a hematological malignancy, which is characterized by aberrant plasma cells (PC) clonal proliferation in the bone marrow (BM) or, less commonly, in extramedullary lesions. Monoclonal proteins (IgG, $\operatorname{IgA}, \quad \operatorname{IgD}, \quad \operatorname{IgE}$ immunoglobulin) and/or monoclonal immunoglobulin free light chains (FLC) are produced by cells of $\mathrm{MM}^{(2)}$.

The existence of $10 \%$ or more clonal plasma cells on BM examination or a biopsy-proven plasmacytoma, in addition to one of the following, is required for diagnosis: renal failure, hypercalcemia, anemia, or lytic bone lesions ${ }^{(3)}$.

Multiparametric flowcytometry (MFC) is a reliable method for MM diagnosis, classification, and prognosis. To rule out other cells contaminating PC gating, MFC uses a combination of high to moderate coexpression of $\mathrm{CD} 38$ and $\mathrm{CD} 138$ with weak and/or negative CD45 to identify MM cells in BM ${ }^{(4)}$.

In relapsed or resistant cases CD138 is lost in varying degrees, while CD38 expression is reduced. Moreover, CD138 has a poorer stability at low temperatures, necessitating the addition of a new stable and reliable marker in the FC panel of $\mathrm{MM}^{(5)}$.

T-lymphocyte surface antigen Ly-9 (CD229) and SLAM family member 7 , which is a protein encoded by the SLAMF7 (CD319) are members of the signaling lymphocyte activating molecule (SLAM) that have been found on the surface of malignant PCs in varying degrees ${ }^{(5,6)}$.

Despite this variable expression of these markers on MM cells, they are effective gating indicators in FC for accurate diagnosis, and they have been studied as probable therapeutic targets for MM. CD319 knockdown may affect the development of $\mathrm{MM}^{(7)}$.

The protooncogene c-Maf is an avian retrovirus cellular homolog transforming gene linked to $(14 ; 16)$ translocation. By boosting integrin 7 adherence to BM stroma, it promotes cell cycle progression and vascular endothelial growth factor production, and it may play a role in MM pathogenesis ${ }^{(\mathbf{8 , 9})}$.

Overexpression of c-Maf may have a prognostic significance in the survival of MM. The clinical 
implication of the c-Maf suggest that it could be a target for therapeutic intervention in cancer treatment ${ }^{(\mathbf{1 0})}$. The detection of the transcription factor c-Maf using immunohistochemistry (IHC) can be employed as a tool for MM prognostic analysis ${ }^{(11)}$.

Furthermore, CD319 promoted c-Maf targeted cyclin D2-dependent proliferation of MM cell. This suggested that CD319 and c-Maf may have a role in MM pathogenesis and prognosis ${ }^{(\mathbf{8})}$. CD319 and CD229 studies are scattered and limited to a small patients cohorts in clinical trials, implying that more study is needed $^{(6,7)}$.

The aim of this study was to evaluate the clinical importance of CD229, CD319, and c-Maf overexpression in MM patients, as well as their impact on the treatment outcome.

\section{PATIENTS AND METHODS}

Sixty one (61) newly diagnosed multiple myeloma patients were involved in this prospective cohort study. They were referred to Clinical Hematology Unit, Clinical Pathology and Pathology Departments, Faculty of Medicine, Zagazig University Hospitals in the period between May 2017 to November 2019. Multiple myloma diagnosis was based on the WHO 2016 criteria and Rajkumar's ${ }^{(12)}$ method.

All included patients underwent the followings: Complete medical history, clinical examination, and routine laboratory investigations. BM aspirate and biopsy samples were obtained from all the MM patients for morphological assessment, immunophenotyping, and immunohistochemistry.

BM aspirate was collected in EDTA tubes (1.5 $\mathrm{mg} / \mathrm{ml}$ ) and immunophenotypic analysis was performed within 6 hours after collection. Leishman stained films were also examined morphologically and bone marrow plasma cells (BMPCs) were counted.

\section{Flow cytometric Analysis:}

For MFC analysis $100 \mu \mathrm{l}$ of blood count-adjusted anticoagulated bone marrow sample was incubated with monoclonal antibodies at room temperature in the dark for 15 minutes. Red blood cells were lysed and washed twice with phosphate buffered saline (PBS) after incubation, then, the supernatant was discarded, and the cell pellet was suspended in PBS.

The acquisition of 50,000 nucleated cells and subsequent analyses were performed using a FACSCalibur Becton Dickinson flow cytometer (BD Bioscience, San Jose, CA, USA). CellQuest software BD Bioscience was used for analysis. A specific 4 colour monoclonal panels were used CD138 PerCP, CD56 FITC, CD20 PE, CD45 APC CD19 PerCP, CD20 FITC, CD200PE, CD229PE, CD319PE, $\kappa$, and $\lambda$ lightchain Ig (PE and FITC respectively) to confirm clonality, then lysis solution was added to the tube vortexer immediately for 10 minutes, followed by incubation for 10 minutes at room temperature in the dark. After washing with PBS, cells were fixed by the adding $500 \mu \mathrm{L}$ of $1 \%$ PBS-formaldehyde. The cells were considered positive for a marker if more than $20 \%$ of cells expressed that marker according to WHO 2016. CD138 and side scatter were used to identify plasma cells, and then CD138+ gated cells were tested for CD56, CD19, and CD45.

Plasma cells were gated using CD38 and side scatter, and CD38+ cells with low side scatter were regated for negative or intermediate CD45 expression, while neoplastic plasmalls revealed CD19- and CD56+ expression ${ }^{(13)}$.

CD138 PerCP, CD38 FITC, CD45 APCCD56FITC were among the monoclonal antibodies employed. To confirm clonality, use CD19 PerCP, CD20PE, CD200PE, CD229PE, CD319 PE, intracellular, and light chain Ig (PE and FITC respectively).

\begin{tabular}{|l|l|l|l|}
\hline FITC & PE & PerCP & APC \\
\hline CD38 & CD20 & CD138 & CD45 \\
\hline CD56 & CD319 & CD138 & CD45 \\
\hline CD38 & CD229 & CD19 & CD45 \\
\hline NK & $\lambda$ & CD19 & CD45 \\
\hline CD38 & CD200 & CD19 & CD45 \\
\hline
\end{tabular}

\section{Immunohistochemical Analysis:}

Biopsies from BM were collected from all included patients. Anti CD138 antibody (1:100, Serotec, Oxford, UK), was used for plama cells labelling.vThen immunohistochemical analysis was carried out using anti c-Maf monoclonal antibody (Vector laboratories, Burlingame, CA, USA).

After that, labeling with biotin-streptavidinhorse peroxidase and Nova Red substrate development was performed. We used skin biopsies as positive control for c-Maf immunostaining, while negative control was performed by replacing the primary antibody with non-immune mouse serum. c-Maf was identified as nuclear staining. It was considered positive if more than $20 \%$ of plasma cells in the BM showed positive nuclear expression ${ }^{(11)}$.

\section{Therapeutic regimens:}

All patients in this study were treated by combination regimens of immunomodulatory drugs (IMiDs) and proteasome inhibitors (PIs) for 4 cycles before response evaluation, such as: Bortezomib/cyclophosphamide/ dexamethasone (VCD) (14) (Cyclophosphamide $300 \mathrm{mg} / \mathrm{m}^{2}$ days 1, 8, 15 and 22; bortezomib $1.3 \mathrm{mg} / \mathrm{m}^{2}$ subcutaneously on days $1,8,15$, 22 ; dexamethasone $40 \mathrm{mg}$ orally on days $1-4,9-12,17-$ 20, 28; for each cycle), Bortezomib/ thalidomide/dexamethasone (VTD) ${ }^{(15)}$ (Bortezomib 1.3 $\mathrm{mg} / \mathrm{m}^{2}$ subcutaneously days $1,8,15,22$; thalidomide $100 \mathrm{mg}$ orally days $1-21$; dexamethasone $40 \mathrm{mg}$ on days 1-4, 8-11, 21; for each cycle), Bortezomib/lenalidomide/dexamethasone (VRd) (16) (Bortezomib $1.3 \mathrm{mg} / \mathrm{m}^{2}$ subcutaneously days $1,8,15$, 22; lenalidomide $25 \mathrm{mg}$ orally days 1-14; dexamethasone $40 \mathrm{mg}$ orally on days 1, 8, 15, 22; for 
each cycle). Almost all of our patients received biphosphonate therapy due to bone lesions.

\section{Therapy outcome criteria:}

Our cases were re-evaluated by $\mathrm{CBC}$, serum B2 microglobulin, serum protein electrophoresis and immunofixation, BMA, and BM biopsy after the fourth cycle. Then, using revised IMW Gresponse criteria ${ }^{(17)}$ and the National Comprehensive Cancer Network $\mathrm{NCCN}^{(18)}$ to assess treatment response.

\section{Ethical consent:}

An approval of the study was obtained from Zagazig University Academic and Ethical Committee.

Every patient signed an informed written consent for acceptance of sharing in the study. This work has been carried out in accordance with The Code of Ethics of the World Medical Association (Declaration of Helsinki) for studies involving humans.

\section{Statistical analysis}

Data analysis was performed by IBM SPSS 23.0 for windows (SPSS Inc., Chicago, IL, USA) and NCSS 11 for windows (NCSS LCC., Kaysville, UT, USA). The quantitative data expression was as mean \pm standard deviation (SD). While qualitative data expression was as frequency and percentage.

We performed the following tests: Independent sample t-test for normally distributed data, while for not normally distributed data we used Mann-Whitney test. For analysis of qualitative data, we used Chi-square and Fisher exact test.

ROC curve analysis was uded for data validity. All tests were two-tailed. A p-value $<0.05$ was considered significant and $\mathrm{p}$-value $<0.001$ was considered as highly significant.

\section{RESULTS}

The demographic and basic characteristics of the studies patients are shown in table 1 .

Table (1): Demographic and basic characteristics of the MM patients

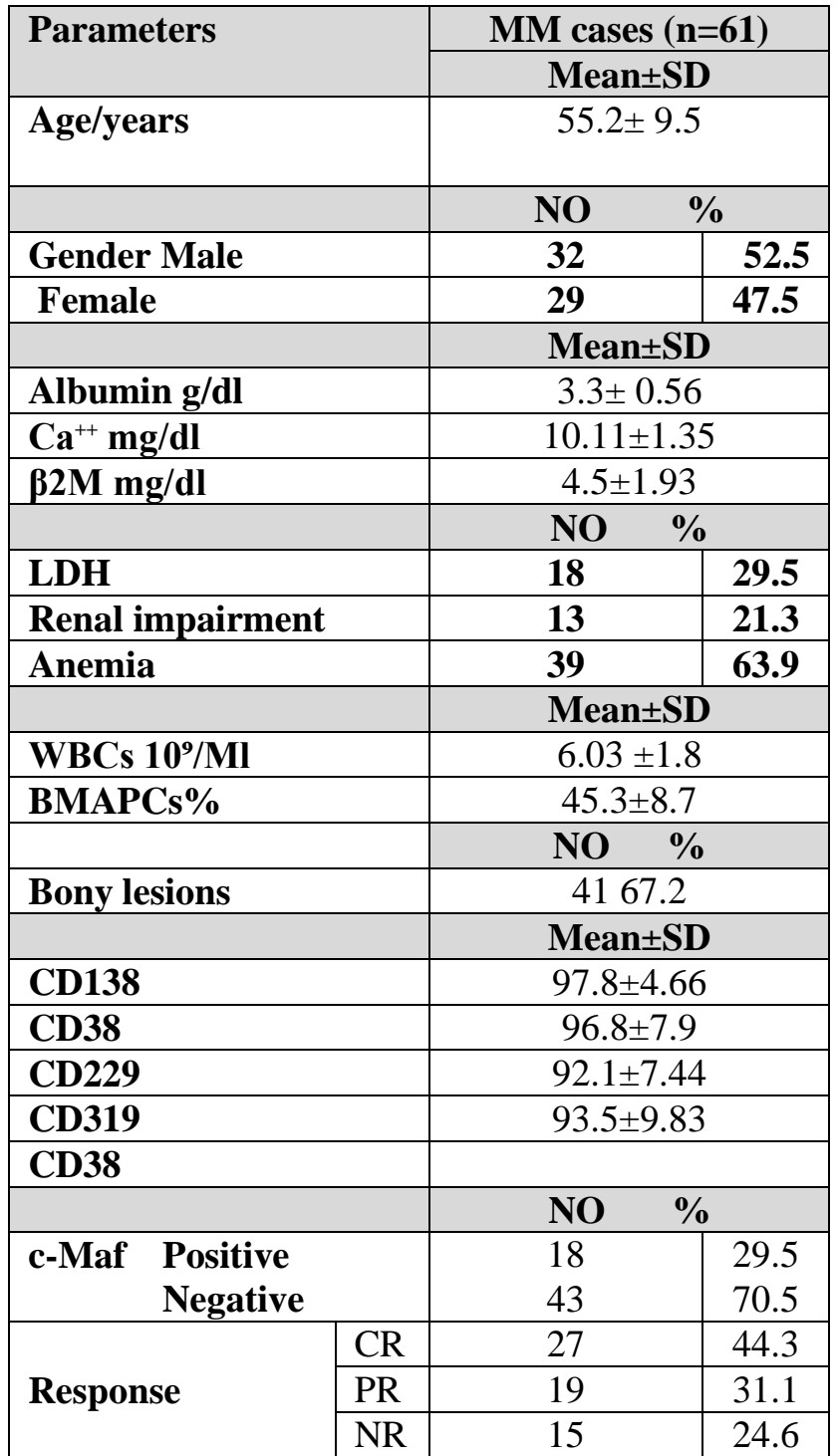

Ca++: calcium; B2M: B2 microglobulin; CR: complete remission; PR: partial response: NR: no response.

Correlation between expression of CD229 \& characteristic data and response to treatment: CD229 expression was high $(\geq 93.9 \%)$ in 34 patients. In these patients serum $\mathrm{Ca}, \beta_{2} \mathrm{M}, \mathrm{CD} 38, \mathrm{CD} 138$, and CD319 expression and percentage of c-Maf positive cases were significantly higher compared to those with low CD229 expression. There was a statistically significant association between expression of CD229 and response to treatment. As low expression of CD229 was associated with a significant better response to treatment (Table 2 and Figures 1,2). 
Table (2): Correlation between expression of CD229 \& characteristic data and response to treatment in MM patients

\begin{tabular}{|c|c|c|c|c|c|c|}
\hline \multirow{2}{*}{\multicolumn{2}{|c|}{ Parameters }} & \multicolumn{2}{|c|}{$\begin{array}{c}\text { High } \\
\mathrm{n}=34(55.7 \%)\end{array}$} & \multicolumn{2}{|c|}{$\begin{array}{c}\text { Low } \\
\mathrm{n}=27(44.3 \%)\end{array}$} & \multirow[t]{2}{*}{ P-Value } \\
\hline & & \multicolumn{4}{|c|}{ Mean \pm SD } & \\
\hline \multicolumn{2}{|l|}{ Age/years } & \multicolumn{2}{|c|}{$54.7 \pm 8.9$} & \multicolumn{2}{|c|}{$55.9 \pm 10.5$} & 0.63 \\
\hline & & NO & $\%$ & $\mathbf{N}$ & $\%$ & P-value \\
\hline \multirow[t]{2}{*}{ Gender } & Male & 21 & 61.8 & 11 & 40.7 & \multirow[t]{2}{*}{0.10} \\
\hline & Female & 13 & 38.2 & 16 & 59.3 & \\
\hline & \multicolumn{4}{|c|}{ Mean \pm SD } & \\
\hline & $\begin{array}{l}\text { Albumin g/dl } \\
\mathbf{C a}^{++} \mathbf{g} / \mathbf{d l}\end{array}$ & \multicolumn{2}{|c|}{$3.34 \pm 0.56$} & \multicolumn{2}{|c|}{$3.25 \pm 0.56$} & 0.54 \\
\hline $\mathrm{Ca}^{++} \mathrm{g} / \mathrm{dl}$ & & \multicolumn{2}{|c|}{$11.08 \pm 1.51$} & \multicolumn{2}{|c|}{$8.71 \pm 0.95$} & $<0.001 *$ \\
\hline \multicolumn{2}{|l|}{$\beta 2 M ~ m g / d l$} & \multicolumn{2}{|c|}{$8.07 \pm 1$} & \multicolumn{2}{|c|}{$3.49 \pm 0.99$} & $<0.001 * *$ \\
\hline \multicolumn{2}{|c|}{ (2) } & $\mathbf{N}$ & $\%$ & $\mathbf{N}$ & $\%$ & P-value \\
\hline \multicolumn{2}{|c|}{ High LDH } & 9 & 26.5 & 9 & 33.5 & 0.56 \\
\hline \multicolumn{2}{|c|}{ Renal impairment } & 8 & 23.5 & 5 & 18.5 & 0.64 \\
\hline \multicolumn{2}{|l|}{ Anemia } & 19 & 55.9 & 20 & 74.1 & 0.14 \\
\hline & & \multicolumn{4}{|c|}{ Mean \pm SD } & \\
\hline \multicolumn{2}{|c|}{ WBCs $10^{9} / \mathrm{ML}$} & \multicolumn{2}{|c|}{$60.56 \pm 1.97$} & \multicolumn{2}{|c|}{$60.01 \pm 1.61$} & 0.91 \\
\hline \multirow{2}{*}{\multicolumn{2}{|c|}{ BMA PCs\% }} & \multicolumn{2}{|c|}{$44.4 \pm 2.3$} & \multicolumn{2}{|c|}{$46.3 \pm 6.8$} & 0.69 \\
\hline & & $\mathbf{N}$ & $\%$ & $\mathbf{N}$ & $\%$ & P-value \\
\hline Bony lesio & & 21 & 61.8 & 20 & 74.1 & 0.31 \\
\hline & & & Mea & & & \\
\hline CD138 & & & & & & $0.04 *$ \\
\hline CD38 & & & & & & $<0.001 * *$ \\
\hline CD319 & & & & & & 0.005 \\
\hline & & $\mathbf{N}$ & $\%$ & $\mathbf{N}$ & $\%$ & \\
\hline & Positive & 14 & 41.2 & 4 & 14.8 & $0025 *$ \\
\hline c-Mat & Negative & 20 & 58.8 & 23 & 85.2 & $0.025^{*}$ \\
\hline & $\mathbf{C R}$ & 11 & 32.4 & 16 & 59.3 & $0.04 *$ \\
\hline Response & PR & 9 & 26.5 & 10 & 37.0 & 0.38 \\
\hline & NR & 14 & 41.2 & 1 & 3.7 & $<0.001^{* *}$ \\
\hline
\end{tabular}

$*$ : significant, **HS: high significant
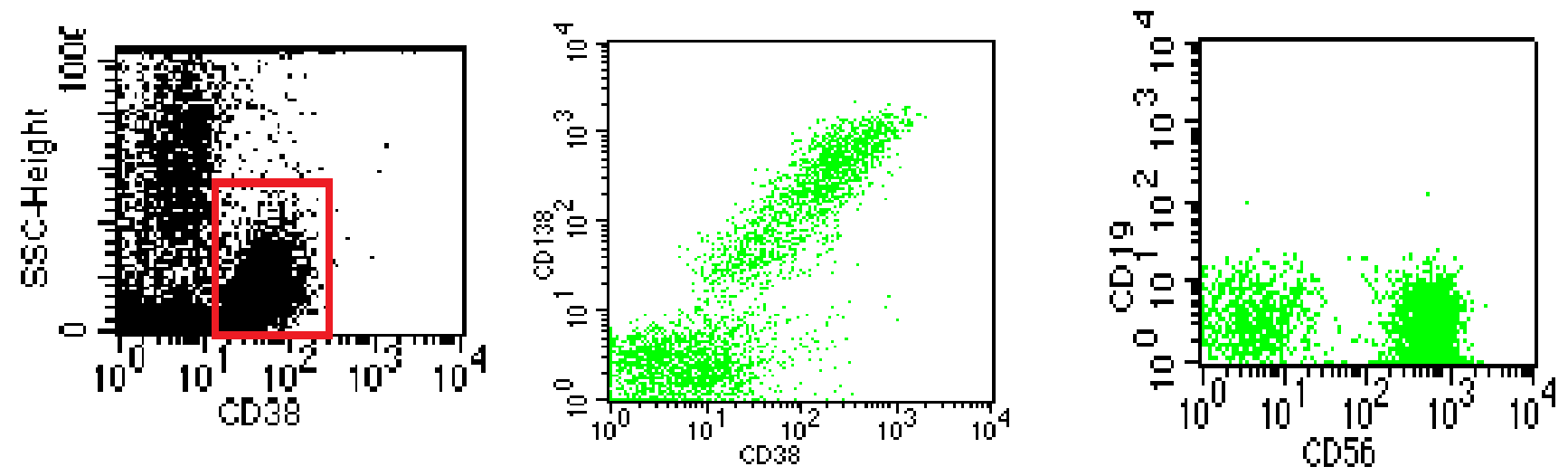

Figure (1): Illustrating example of the expression profile of malignant plasma cells (mPCS), SSC/CD38, coexpression of CD38/CD138, + CD56 with - ve CD19 on (mPCs). 

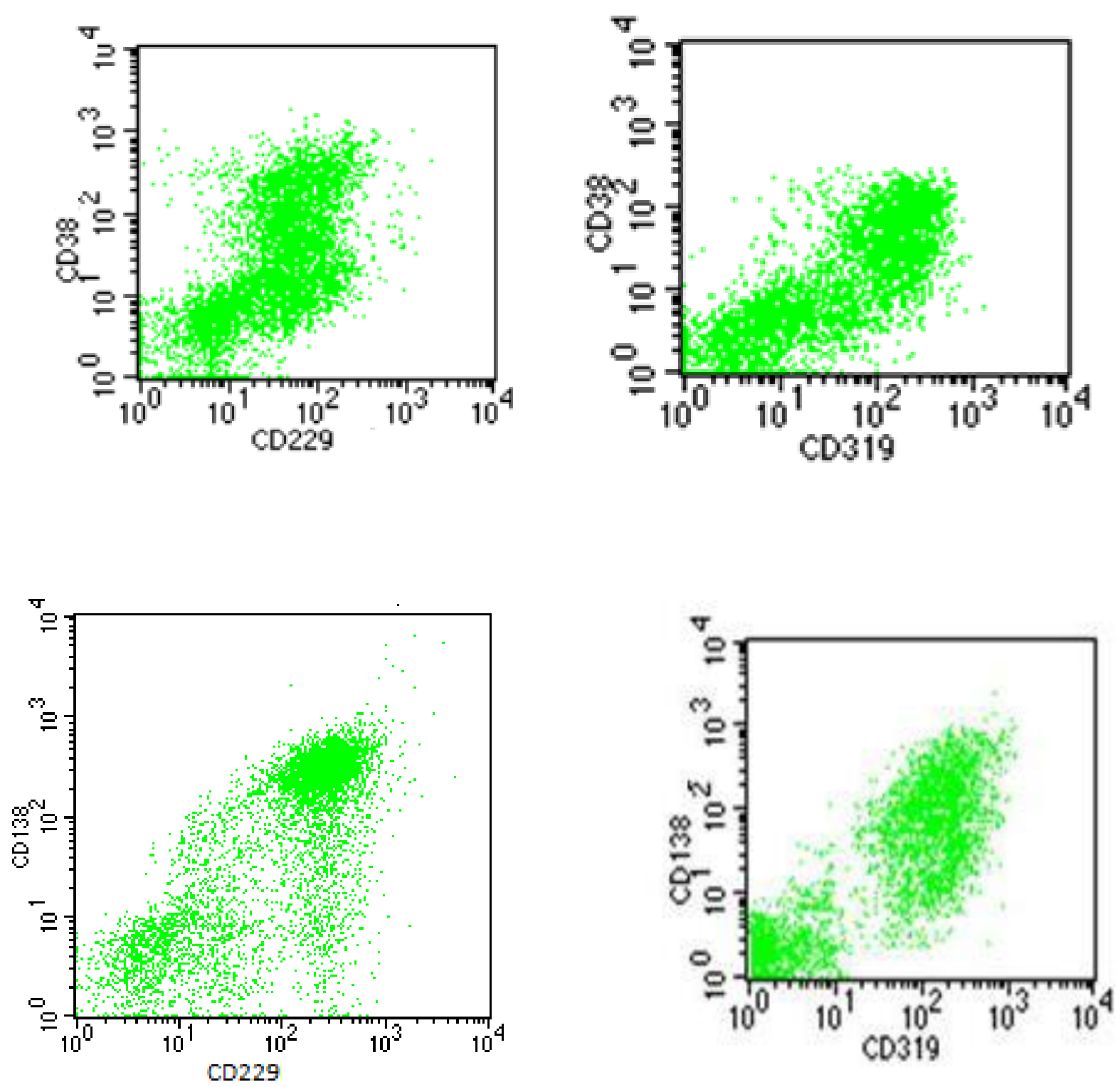

Figure (2): Illustrating example of the expression profile of CD229 and CD319 on BM malignant plasma (MPCs) cells population. They are plotted against CD319/CD38,CD229/CD38,CD138/CD229 and CD138/CD319.

\section{Correlation between expression of $\mathrm{CD319} \&$ characteristic data and response to treatment:}

CD319 expression was high ( $\geq 95.6 \%$ ) in 29 patients (47.5\%). WBCs count, seurm $\mathrm{Ca}, \beta_{2} \mathrm{M}, \mathrm{CD} 229, \mathrm{CD} 38$, and CD138 expression and percentage of c-Maf positive cases were significantly higher in MM patients with high CD319 expression. Also a significant better response to treatment was detected in MM patients showing low expression of CD319 (Table 3). 
https://ejhm.journals.ekb.eg/

Table (3): Correlation between expression of CD319 \& characteristic data and response to treatment in MM patients

\begin{tabular}{|c|c|c|c|c|c|c|}
\hline \multirow{2}{*}{\multicolumn{2}{|c|}{ 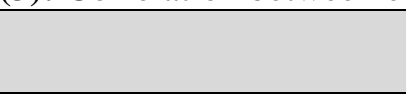 }} & \multicolumn{2}{|c|}{ High, $n=29(47.5 \%)$} & \multicolumn{2}{|c|}{ Low. $n=32(52.5 \%)$} & P-Value \\
\hline & & \multicolumn{4}{|c|}{ Mean \pm SD } & \\
\hline \multicolumn{2}{|l|}{ Age/ years } & \multicolumn{2}{|c|}{$54.6 \pm 9.18$} & \multicolumn{2}{|c|}{$55.8 \pm 9.81$} & 0.63 \\
\hline & & $\mathrm{N}$ & $\%$ & $\mathrm{~N}$ & $\%$ & P-Value \\
\hline \multirow{2}{*}{ Gender } & Male & 19 & 65.5 & 13 & 40.6 & \multirow{2}{*}{0.08} \\
\hline & Female & 10 & 34.5 & 19 & 59.4 & \\
\hline & \multicolumn{4}{|c|}{ Mean \pm SD } & P-Value \\
\hline & Albumin g/dl & \multicolumn{2}{|c|}{$3.33 \pm 0.54$} & \multicolumn{2}{|c|}{$3.2 \pm 0.58$} & 0.71 \\
\hline $\mathrm{Ca}^{++} \mathrm{mg} / \mathrm{dl}$ & & \multicolumn{2}{|c|}{11.021 .7} & \multicolumn{2}{|c|}{$9.12 \pm 1.22$} & $<0.001$ \\
\hline \multirow{2}{*}{\multicolumn{2}{|c|}{$\beta_{2} M$ mg/dl }} & \multicolumn{2}{|c|}{$7.6 \pm 3.75$} & \multicolumn{2}{|c|}{$4.6 \pm 1.86$} & 0.05 \\
\hline & & $\mathbf{N}$ & $\%$ & $\mathbf{N}$ & $\%$ & P-Value \\
\hline \multicolumn{2}{|l|}{ High LDH } & 9 & 31.0 & 9 & 28.1 & 0.804 \\
\hline \multicolumn{2}{|c|}{ Renal impairment } & 7 & 24.1 & 6 & 18.8 & 0.61 \\
\hline \multirow{2}{*}{\multicolumn{2}{|c|}{ Anemia }} & 18 & 62.1 & 21 & 65.5 & 0.77 \\
\hline & & \multicolumn{4}{|c|}{ Mean \pm SD } & P-Value \\
\hline \multicolumn{2}{|c|}{ WBCs 10\%/MI } & \multicolumn{2}{|c|}{$66.16 \pm 1.802$} & \multicolumn{2}{|c|}{$55.03 \pm 1.71$} & $0.02 *$ \\
\hline \multirow{2}{*}{\multicolumn{2}{|c|}{ BMAPCs\% }} & \multicolumn{2}{|c|}{$40.97 \pm 2$} & \multicolumn{2}{|c|}{$49.3 \pm 6.8$} & 0.09 \\
\hline & & $\mathbf{N}$ & $\%$ & $\mathbf{N}$ & $\%$ & P value \\
\hline \multicolumn{2}{|c|}{ Bony lesions } & 20 & 69.0 & 21 & 65.6 & 0.78 \\
\hline \multicolumn{2}{|c|}{ Mean \pm SD } & & & & & P-Value \\
\hline \multicolumn{2}{|l|}{ CD138 } & & & & & 0.01 \\
\hline CD38 & & & & & & 0.05 \\
\hline CD229 & & & & & & $<0.001$ \\
\hline & Positive & 17 & 58.6 & 1 & 3.1 & $<00 \cap 1$ \\
\hline c-Maf & Negative & 12 & 41.4 & 31 & 96.9 & 0.001 \\
\hline & $\mathrm{CR}$ & 0 & 0.0 & 27 & 84.4 & $<0.001$ \\
\hline Response & PR & 16 & 55.2 & 3 & 9.4 & $<0.001$ \\
\hline & NR & 13 & 44.8 & 2 & 6.2 & $<0.001$ \\
\hline
\end{tabular}

Correlation between expression of $\mathrm{C}$-Maf $\&$ characteristic data and response to treatment:

C-Maf was positive in 18 patients (29.5\%). WBCs count, serum Ca, $32 \mathrm{M}, \mathrm{CD} 229, \mathrm{CD} 38, \mathrm{CD} 138$ and CD319 were significantly higher in c-Maf positive cases. Our study detected that there was significantly less complete response rate and more non responding rate noticed in c-Maf positive cases (Table 4 and Figure 3).

Table (4): Correlation between expression of C-Maf \& characteristic data and response to treatment in MM patients

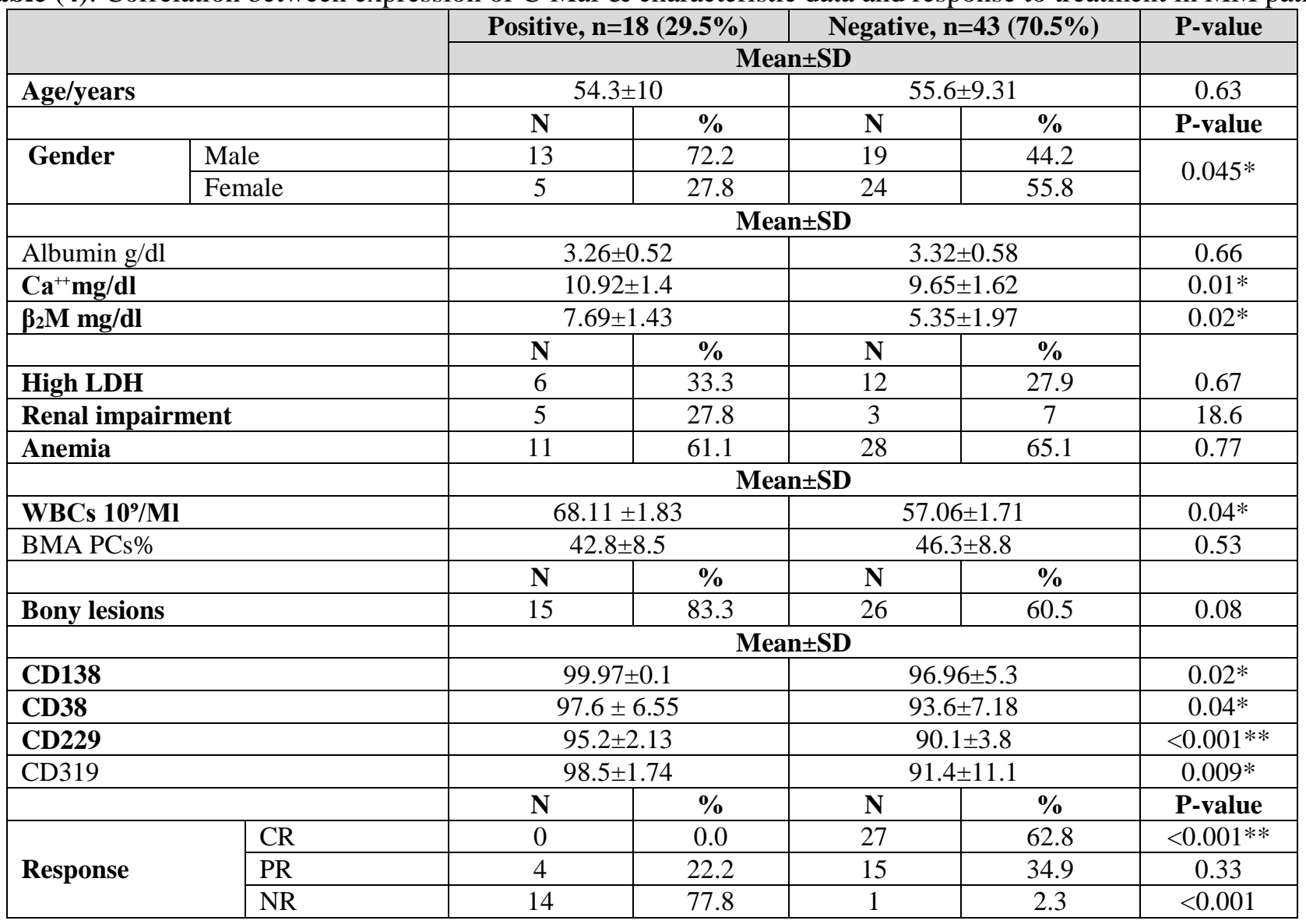

*: significant, **HS: high significant 

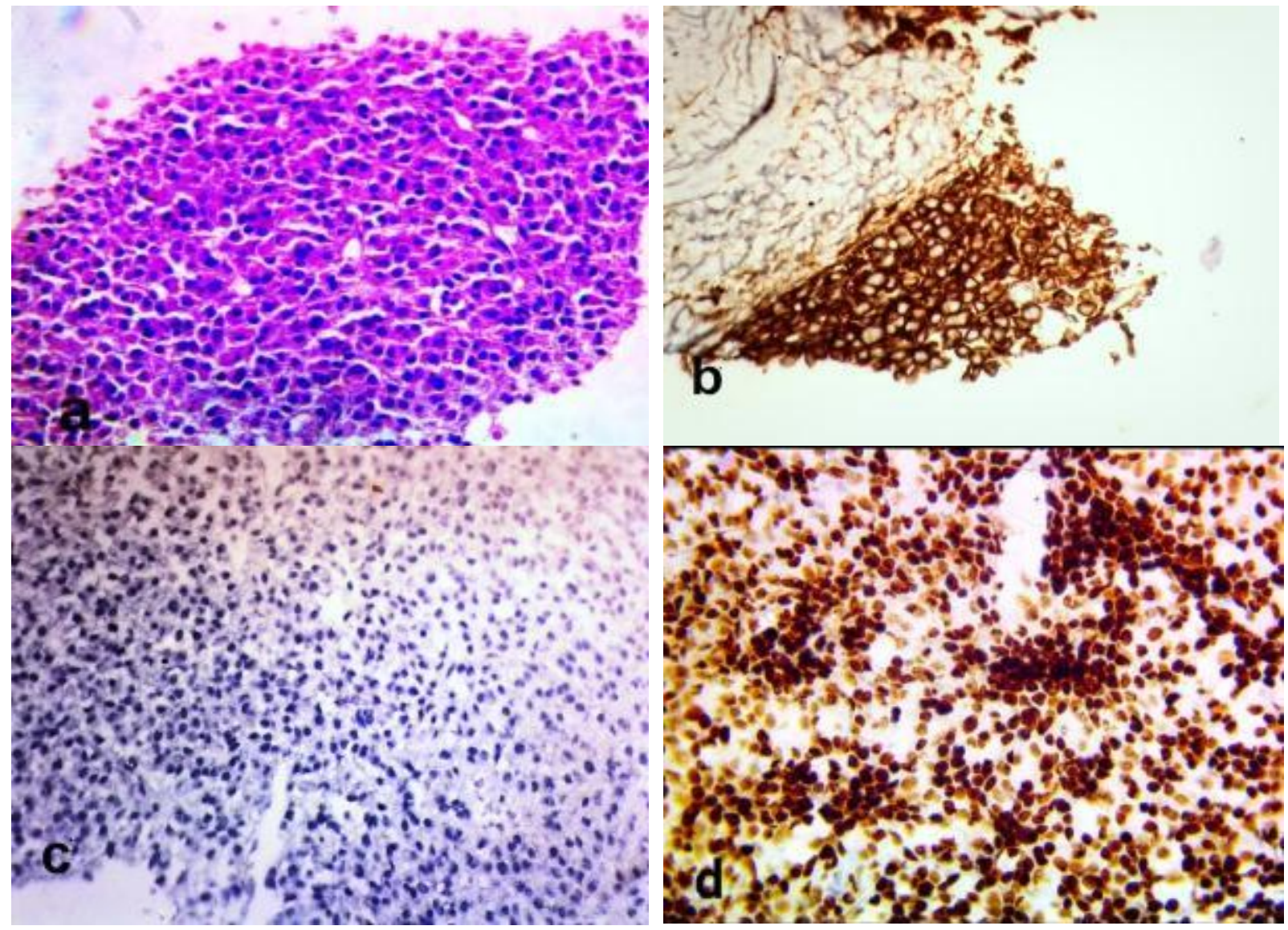

Figure (3): Bone marrow biopsy from MM patients showing (a) Cells with plasmacytoid features (H and E x200) (b) Positive CD138 membranous immunohistochemical expression (IHC x200) (c) Negative c-Maf immunohistochemical expression (IHC x200) (d) Positive c-Maf nuclear immunohistochemical expression (IHC x 200).

Validity of CD229 and CD319 as prognostic markers for plasma cells identification

On studying the validity of CD229 and CD319 as prognostic markers for plasma cells, CD229 the cut off value was 93.95 (Table 5 and Figure 4).

Table (5): Validity of CD229 and CD319 as prognostic markers for plasma cells identification

\begin{tabular}{|l|c|c|c|c|c|c|c|c|}
\hline & AUC & P & Cut off & Sensitivity & Specificity & PPV & PPN & Accuracy \\
\hline CD229 & 0.734 & 0.007 & 93.95 & 88.9 & 76.7 & 61.5 & 94.3 & 80.3 \\
\hline CD319 & 0.839 & $<0.001$ & 95.6 & 94.5 & 86.7 & 73.9 & 97.4 & 88.5 \\
\hline
\end{tabular}

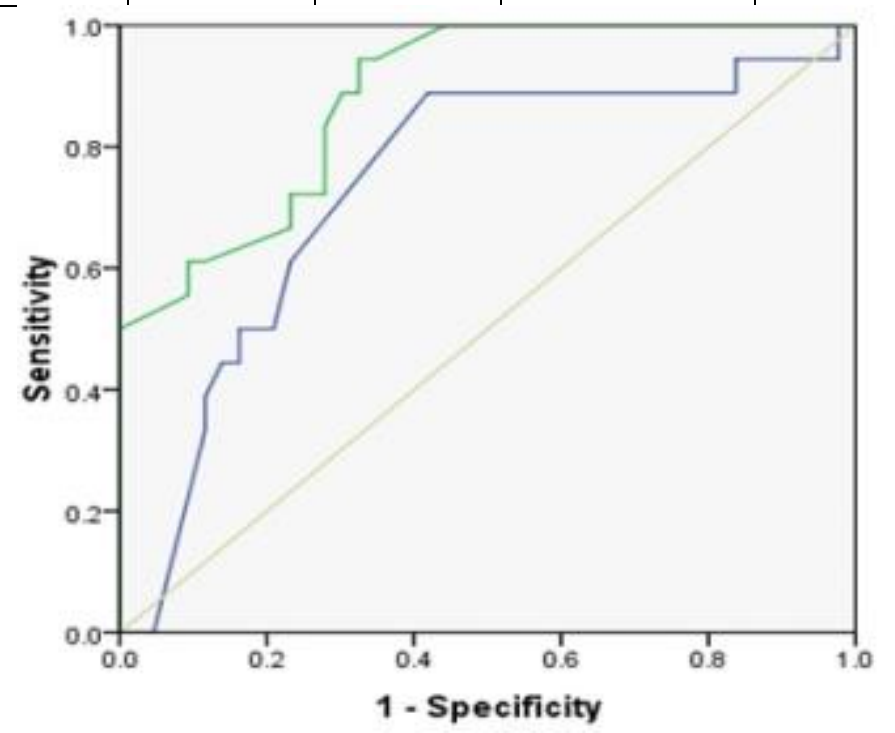

Source of the Curve

- $\mathrm{CD} 229$

Reference Line

Figure (4): Receiver operating characteristics (ROC) curve for the validity of CD229 and CD319 as prognostic markers for MM. 


\section{DISCUSSION}

Flow cytometry is a vital tool for the MM daiagnosis through the identification of specific surface markers (4). Plasma cells were identified as CD138+/CD38+ events after an initial gate with low SSC in the CD45/SSC cytogram ${ }^{(\mathbf{1 9})}$. CD138 shows a variable loss or decreased expression in MM patients with relapsed or progressive disease ${ }^{\mathbf{2} 2)}$. The use of antiCD38 or anti-CD138 in the treatment of MM may limit their value in follow up so more stable markers may be needed $^{(5,19,20)}$.

CD229 is a more constant overexpressed antigen on MM cells ${ }^{(6)}$. CD319 is widely expressed on malignant plasma cells playing a major role in MM pathogenesis ${ }^{(21)}$. Furthermore, CD319 promoted the transcription factorc-Maf targeted cyclin D2-dependent MM cells proliferation, so c-Maf may be used in the prognostic analysis of MM ${ }^{(22)}$. This provided direct evidence for the role of CD319 and c-Maf in myeloma pathogenesis and prognosis ${ }^{(23)}$. Studies about CD319 and CD229 are dispersed and limited to a small patients cohorts in clinical trials, suggesting the need for more research $^{(9)}$. c-Maf expression, MM characteristics and prognosis had not been widely explored ${ }^{(24)}$.

This work was designed to evaluate the clinical implication of CD319, CD229, and c-Maf overexpression on treatment response in multiple myeloma patients.

In this study, we found that both CD229 and CD319 were widely expressed on MM cells. This finding was in agreement with Olson et al. ${ }^{(25)}$, who verified that CD229 has a wide, strong, and homogenous expression on malignant PCs. These results are also supported by Ishibashi et al. ${ }^{(7)}$ and Sriram et al. ${ }^{\text {(26) }}$ who reported that CD319 was highly expressed cells of MM and correlated with progression of the disease.

We found that the mean values of CD38, CD138 expression, and the percentage of c-Maf positive cases were higher significantly in MM patients with high expression of CD229 and CD319. We found also that CD229 and CD319 were significantly correlated with CD138and CD38. This wide expression of CD229 and CD319 on MM cells and their significant correlation with CD38 and CD138 may suggest their usefulnessas as an alternative diagnostic marker for MM. This is supported by Pojero et al. ${ }^{(5)}$ who compared the classic markers CD138 and CD38 with these possible alternative markers and found that CD229 and CD319 have been identified as a candidate to potentially replace the classic markers especially in follow up cases treated by specific anti-CD138 and anti-CD38 monoclonal antibodies and also in relapsed or resistant cases. The efficacy of using CD229 and CD319 as alternative markers for the diagnosis of $\mathrm{MM}$ is also supported by Sriram et al. ${ }^{(26)}$ who suggested the utility of CD319 as a new diagnostic marker in MM. This suggestion is also supported by Ghogale $\boldsymbol{e t} \boldsymbol{a l} \mathbf{~}^{(\boldsymbol{( 6 )}}$ who stated that CD229 is a highly stable immunophenotypic marker showing strong and homogenous expression in PCs. Hand by hand with these results is the study of Muccio et al. ${ }^{\mathbf{( 1 9 )}}$ who suggested that CD319 and CD229 can be added to the panel for PC identification and MRD assessment.

In this work, c-Maf expression by immunohistochemistry was detected to be positive in $29.5 \%$ of MM patients. Our result is supported by Wei et al. ${ }^{(11)}$ who detected c-Maf overexpressed in $35.5 \%$ of MM cases. We found also a significant difference between c-Maf positive and negative cases as regard CD229 and CD319 suggesting the relationship between these markers and c-Maf oncogene. Tai et al. ${ }^{(8)}$ also stated that the association between CD319 and c-Maf overexpression in $\mathrm{MM}$ is due to transactivation of $\mathrm{c}$ Maf by CD319 augmenting cell proliferation and enhancing interactions between stromal cells and tumors cells.

In this work, as we found that patients with low expression of CD319 and CD229 and c-Maf negative have a better response to treatment, we suggest the predictive significance of these markers. This is supported by Olson et al. ${ }^{(25)}$ who demonstrated the poor prognostic value of CD229 in MM and Radhakrishnan et al. ${ }^{(27)}$ who stated that CD229 is a poor prognostic marker in $\mathrm{MM}$ and its target therapy may eradicate terminally differentiated MM cells and clonotypic MMpropagating cells. Radhakrishnan et al. ${ }^{(27)}$ also stated that targeting CD229 can treat the MM-resistant cases and potentially providing long-lasting responses and Postelnek et al. ${ }^{(28)}$, who demonstrated the prognostic role of CD319, reported that a high concentration of CD319 in the microenvironment of BM may suppress elotuzumab effects in MM patients. Xie et al. ${ }^{(29)}$ supported these results also by reporting that CD319 is included in tumor proliferation in MM inducing tumor cell growth and it is linked to poor prognosis in MM.

In our study, the percentage of sensitivity, specifity and accuracy of CD319 was higher than CD229 as a prognostic marker for plasms cells. This is in disagreement with Pojero et al. ${ }^{(5)}$ who detected that CD229 may be more reliable marker than CD319 to replace CD38 for plasma cells identification.

One of the limitations in our study is that our study aimed to estimate the correlation between positive expression of c-Maf, CD229,CD319 overexpression and response to treatment. Our study did not estimate the patients' survival.

\section{CONCLUSION}

The conclusion drawn from this work is that both CD229 and CD319 were significantly overexpressed on MM cells and correlated significantly with CD138 and CD38, suggesting their utility as alternative markers for diagnosis and follow-up of MM. CD229, CD319 and cMaf overexpression were associated with a significant poor response to therapy. This may suggest the significance of these markers as predictors for treatment response. 


\section{RECOMMENDATION}

Further large-scale prospective study as well as longer follow-up periods required to estimate the survival outcome in those patients with CD229, CD319 and c-Maf overexpression. We hope in the future to shed the light on the possibility of therapy targeting CD229. CD319 and c-Maf.

Financial support and sponsorship: Nil.

Conflict of interest: Nil.

\section{REFERENCES}

1. Anderson K, Alsina $M$, Bensinger $W$ et al. (2011): National Comprehensive Cancer network. NCCN clinical practice guidelines in oncology: multiple myeloma. J Natle Compr Canc Netw., (9):908-42.

2. Rajkumar S (2016): Updated diagnostic criteria and staging system for multiple myeloma. Am Soc Clin Oncol Educ Book, 35:418-423.

3. Vincent $\mathbf{S}$ (2016): Updated diagnostic criteria and staging system for multiple myeloma. Am Soc Clin Oncol Educ Book, 35:418-23.

4. Rawstron A, Orfao A, Beksac M et al. (2008): Report of the European Myeloma Network on multiparametric flow cytometry in multiple myeloma and related disorders. Haematologica, 93(3):431-438.

5. Pojero F, Flores-Montero J, Sanoja L et al. (2016): EuroFlow group. Utility of CD54, CD229, and CD319 for the identification of plasma cells in patients with clonal plasma cell diseases. Cytometry B Clin Cytom, 90(1):91-100.

6. Ghogale $S$, Tembhare $P$, Tauro $W$ et al. (2018): Subramanian. evaluation of CD229 as a new alternative 5evaluation of CD229 as a new alternative plasma cell gating marker in the flow cytometric immunophenotyping of monoclonal gammopathies, cytometry part B. Clinical Cytometry, 94(3):509-519.

7. Ishibashi M, Soeda S, Sasaki M et al. (2018): Clinical impact of serum soluble SLAMF7 in multiple myeloma. Oncotarget, 9(78):34784-34793.

8. Tai Y, Soydan E, Song W et al. (2009): CS1 promotes multiple myeloma cell adhesion, clonogenic growth, and tumorigenicity via c-Maf-mediated interactions with bone marrow stromal cells, Blood, 113(18):4309-18.

9. Rollig C, Knop S, Bornhauser M (2015): Multiple myeloma. Lancet, 385(9983):2197-208.

10. Chang H, Qi Q, Xu W et al. (2007): c-Maf nuclear oncoprotein is frequently expressed in multiple myeloma. Leukemia, 7:1572-4.

11. Wei G, Li J, Han J et al. (2017): Clinical implications of cmaf expression in plasma cells from patients with multiple myeloma. Exp Hematol Oncol., 6:16-23.

12. Rajkumar S (2018): Multiple myeloma: 2018 update on diagnosis, risk-stratification, and management. Am J Hematol., 93(8):981-1114.

13. Chatterjee G, Gujral S, Subramanian P et al. (2017): Clinical relevance of multicolour flow cytometry in plasma cell disorders. Indian J Hematol Blood Transfus, 33(3):303315.

14. Reeder C, Reece D, Kukreti V et al. (2009): Cyclphosphamide, bortezomib and dexamethasone induction for newly diagnosed multiple myeloma: high response rates in aphase II clinical trial. Leukemia, 23(7):1337-41.

15. Cavo M, Pantani L, Petrucci M et al. (2012): Bortezomibthalidomide-dexamethasone is superior to thalidomidedexamethasone as consolidation therapy after autologous hematopoietic stem cell transplantation in patients with newly diagnosed multiple myeloma. Blood, 120: 9-19.

16. Attal $M$, Lauwers-Cances V, Hulin C et al. (2009): Linalidomide, bortezomib, and dexamethasone with transplantation for myeloma. N Engle J Med., 376(14):13111320.

17. Kumar S, Paiva B, Anderson $\mathrm{K}$ et al. (2016): IMWG consensus criteria for response and minimal residual disease assesment in multiple myeloma. The Lancet Oncology, (8) 328-346.

18. Kumar S, Callander N, Adekola K et al. (2020): Multiple myeloma, version 3.2021, NCCN Clinical Practice Guidelines in Oncology. J Natl Compr Canc Netw. 18(12):1685-1717.

19. Muccio V, Saraci E, Gilestro M et al. (2016): Multiple myeloma: New surface antigens for the characterization of plasma cells in the era of novel agents. Cytometry B Clin Cyto., 90(1) 81-90.

20. Zuchlinsky D, Calvo K, Kurlander R et al. (2014): Flow cytometric differentiation of abnormal and normal plasma cells in the bone marrow in patients with multiple myeloma and its precursor diseases. Leuk Res., 38(3):371-376.

21. Malaer J, Mathew P (2017): CS1 (SLAMF7, CD319) is an effective immunotherapeutic target for multiple myeloma, Am J Cancer Res., 7(8):1637-1641.

22. Natkunam Y, Tedoldi S, Paterson J et al. (2009): Characterization of c-Maf transcription factor in normal and neoplastic hematolymphoid tissue and its relevance in plasma cell neoplasia. Am J Clin Pathol., 132:361-371

23. Cesana C, Klersy C, Barbarano L et al. (2002): Prognostic factors for malignant transformation in monoclonal gammopathy of undetermined significance and smoldering multiple myeloma. J Clin Oncol., 20:1625-1634.

24. Qiang Y, Shiqiao Y, Faith E (20016): MAF protein mediates innate resistance to proteasome inhibition therapy in multiple myeloma, Blood, 128(25):2919-2930.

25. Olson M, Radhakrishnan S, Luetkens T et al. (2009): The role of surface molecule CD229 in Multiple Myeloma. Clinical Immunology, 204: 69-73.

26. Sriram H, Ghohale S, Subramanian $P$ et al. (2019): Evaluation of CD319 (SLAMF7) as a novel gating marker for plasma cells in Flow cytometric immunophenotyping of MM. Clinical Lymphoma Myeloma and Leuckemia, 19(10):153-158.

27. Radhakrishnan S, Luetkens T, Scherer S et al. (2020): CD229 CART cells eliminate multiple myeloma and tumor propagating cells without fratricide. Nature Communications, 11(1):798-804.

28. Postelnek J, Neely R, Robbins M et al. (2016): Development and validation of electrochemiluminescence assays to measure free and total electrochemiluminescence assays to measure free and total sSLAMF7 in human serum in the absence and presence of elotuzumab. AAPS J., 18(4)989-999.

29. Xie Z, Gunaratne J, Cheong L et al. (2013): Plasma membrane proteomics identifies biomarkers associated with MMSET overexpression in $\mathrm{T}(4 ; 14)$ multiple myeloma. Oncotarget., 4(7)1008-1018. 